\title{
Game Theory for Cyber Deception
}

\author{
A Tutorial \\ Quanyan Zhu \\ New York University \\ Brooklyn, New York \\ qz494@nyu.edu
}

\begin{abstract}
Deceptive and anti-deceptive technologies have been developed for various specific applications. But there is a significant need for a general, holistic, and quantitative framework of deception. Game theory provides an ideal set of tools to develop such a framework of deception. In particular, game theory captures the strategic and self-interested nature of attackers and defenders in cybersecurity. Additionally, control theory can be used to quantify the physical impact of attack and defense strategies. In this tutorial, we present an overview of game-theoretic models and design mechanisms for deception and counter-deception. The tutorial aims to provide a taxonomy of deception and counter-deception and understand how they can be conceptualized, quantified, and designed or mitigated. This tutorial gives an overview of diverse methodologies from game theory that includes games of incomplete information, dynamic games, mechanism design theory to offer a modern theoretic underpinning of cyberdeception. The tutorial will also discuss open problems and research challenges that the HoTSoS community can address and contribute with an objective to build a multidisciplinary bridge between cybersecurity, economics, game and decision theory.
\end{abstract}

\section{CCS CONCEPTS}

-Security and privacy $\rightarrow$ Network security; $\bullet$ Mathematics of computing; • Theory of computation $\rightarrow$ Algorithmic game theory and mechanism design;

ACM Reference Format:

Quanyan Zhu. 2019. Game Theory for Cyber Deception: A Tutorial. In HoTSoS '19: Hot Topics in the Science of Security: Symposium and Bootcamp, April 2-3, 2019, Nashville, TN, USA. ACM, New York, NY, USA, 3 pages. https://doi.org/10.1145/3314058.3314067

\section{TUTORIAL DESCRIPTION}

Cyber deception is a technique used to cause human [12, 36] or computer systems $[1,15,20]$ to have false beliefs and behave against their interests. Cyberspace creates unique opportunities for deception. Attackers can leverage information asymmetry to misinform

Permission to make digital or hard copies of part or all of this work for personal or classroom use is granted without fee provided that copies are not made or distributed for profit or commercial advantage and that copies bear this notice and the full citation on the first page. Copyrights for third-party components of this work must be honored. For all other uses, contact the Owner/Author.

HotSoS, April 1-3, 2019, Nashville, TN, USA

(c) 2019 Copyright is held by the owner/author(s)

ACM ISBN 978-1-4503-7147-6/19/04.

https://doi.org/10.1145/3314058.3314067 and mislead the users. For example, phishing is a typical deceptionbased attack that is one of the top threat vectors for cyberattacks. Defenders can also use deception as a way to thwart or deter attacks. For example, techniques such as honeynets $[2,53]$, moving target defense $[19,21,50]$, obfuscation $[8,31,46,48]$, and mix networks [42] have been introduced to create difficulties for attackers to map out the system information.

Successful deception fundamentally depends on the information asymmetry between the deceiver and the deceivee [25, 27, 45, 58]. Deceivers can strategically manipulate the private information to suit their own self-interests. The manipulated information is then revealed to deceivees, who, on the other hand, make decisions based on the information received. It is important for the deceivee to form correct beliefs based on past observations, take into account the potential damage caused by deception, and strategically use the observed information for decision-making.

Modeling deception would formally capture the interactions between an attacker and a defender, provide a quantitative and systematic understanding of deceptions, and develop new technologies and incentive mechanisms to mitigate cyber risks and safeguard the cyberspace. Game-theoretic models are natural frameworks to capture the adversarial and defensive interactions between players [22]. It provides a quantitative measure of the quality of protection with the concept of Nash equilibrium where both defender and an attacker seek optimal strategies, and no one has an incentive to deviate unilaterally from their equilibrium strategies despite their conflict for security objectives. The equilibrium concept also provides a quantitative prediction of the security outcomes of the scenario the game model captures. Recently, game theory has been applied to different sets of security problems, e.g., Stackelberg and signaling games for deception and proactive defenses [7, 32, 50$53,57,61,63]$, (2) network games for cyber-physical security that deals with critical infrastructure protection and information assurance $[5,10,16,23,34,35,37-41]$, dynamic games for adaptive defense $[9,17-19,29,47,49,59,60,62]$, and mechanism design theory for security $[4,6,13,14,43,44,54-56]$.

This tutorial aims to present an overview of game-theoretic methods and show their applications in different scenarios. More specifically, we start with a baseline a multi-stage Bayesian game model with two-sided incomplete information and introduce the analysis of different variants of the baseline that can be used to capture different features of cyber deception. The objective of this tutorial is to introduce diverse methodologies from game theory that include mechanism design, incentive analysis, decision-making under incomplete information, and dynamic games to provide solid underpinnings of cyber deception. 
This tutorial will be structured in the following way. The first part of the tutorial introduces a taxonomy of deception from a gametheoretic perspective [25, 27]. Then, the tutorial introduces the baseline signal games and the analytical methods for two-person deception games [3, 28, 30, 47]. We will present the concept of strategic trust as a defense mechanism that can be designed using the signaling games [11, 15, 24, 26, 33, 37]. The baseline games can be further extended to include side-channel information, system dynamics, and the influence of the third party. The tutorial will also discuss open problems and research challenges that the HoTSoS community can address and contribute. With the objective to build a multidisciplinary bridge between cybersecurity, economics, game and decision theory, this tutorial will review basic concepts and provide an overview of recent advances in the field to HoTSoS community with the hope to establish a community interest in the science of security and cross-disciplinary researches.

The potential audience includes researchers from academia and industry, including $\mathrm{PhD}$ and graduate students. Some background in network security and knowledge of basic optimization and data science is helpful but not necessary.

\section{AUTHOR BIOGRAPHY}

Quanyan Zhu received B. Eng. in Honors Electrical Engineering with distinction from McGill University in 2006, M.A.Sc. from University of Toronto in 2008, and Ph.D. from the University of Illinois at Urbana-Champaign (UIUC) in 2013. After stints at Princeton University, he is currently an assistant professor at the Department of Electrical and Computer Engineering, New York University. He is a recipient of many awards including NSF CAREER Award, NYU Goddard Junior Faculty Fellowship, NSERC Postdoctoral Fellowship (PDF), NSERC Canada Graduate Scholarship (CGS), and Mavis Future Faculty Fellowships. He spearheaded and chaired INFOCOM Workshop on Communications and Control on Smart Energy Systems (CCSES), and Midwest Workshop on Control and Game Theory (WCGT). His current research interests include resilient and secure interdependent critical infrastructures, Internet of Things, cyber-physical systems, game theory, machine learning, network optimization and control. He is a recipient of best paper awards at 5th International Conference on Resilient Control Systems and 18th International Conference on Information Fusion. He has served as the general chair of the 7th Conference on Decision and Game Theory for Security (GameSec) in 2016, the 9th International Conference on NETwork Games, COntrol and OPtimisation (NETGCOOP) in 2018, and the 5th International Conference on Artificial Intelligence and Security (ICAIS 2019) in 2019. Website: http://wp.nyu.edu/quanyan

\section{REFERENCES}

[1] Bodmer, S., Kilger, D. M., Carpenter, G., Jones, J., and Jones, J. Reverse deception: organized cyber threat counter-exploitation. McGraw-Hill New York, 2012.

[2] Carroll, T. E., And Grosu, D. A game theoretic investigation of deception in network security. Security and Commun. Nets. 4, 10 (2011), 1162-1172.
[3] Casey, W., Morales, J. A., Wright, E., Zhu, Q., And Mishra, B. Compliance signaling games: toward modeling the deterrence of insider threats. Computational and Mathematical Organization Theory 22, 3 (2016), 318-349.

[4] Casey, W. A., Zhu, O., Morales, J. A., and Mishra, B. Compliance control: Managed vulnerability surface in social-technological systems via signaling games. In Proceedings of the 7th ACM CCS International Workshop on Managing Insider Security Threats (2015), ACM, pp. 53-62.

[5] Chen, J., Touati, C., And Zhu, Q. A dynamic game analysis and design of infrastructure network protection and recovery. ACM SIGMETRICS Performance Evaluation Review 45, 2 (2017), 128.

[6] Chen, J., ANd Zhu, O. Security as a Service for Cloud-Enabled Internet of Controlled Things under Advanced Persistent Threats: A Contract Design Approach. IEEE Transactions on Information Forensics and Security (2017).

[7] Clark, A., Zhu, Q., Poovendran, R., And Başar, T. Deceptive routing in relay networks. In Decision and Game Theory for Security. Springer, 2012, pp. 171-185.

[8] Farhang, S., Hayel, Y., AND Zhu, Q. Phy-layer location privacy-preserving access point selection mechanism in next-generation wireless networks. In Communications and Network Security (CNS), 2015 IEEE Conference on (2015), IEEE, pp. 263-271.

[9] Farhang, S., Manshaei, M. H., Esfahani, M. N., and Zhu, Q. A dynamic bayesian security game framework for strategic defense mechanism design. In Decision and Game Theory for Security. Springer, 2014, pp. 319-328.

[10] FarooQ, M. J., AND ZHU, Q. Modeling, analysis, and mitigation of dynamic botnet formation in wireless iot networks. IEEE Transactions on Information Forensics and Security (2019).

[11] Fung, C. J., AND ZHu, Q. Facid: A trust-based collaborative decision framework for intrusion detection networks. Ad Hoc Networks 53 (2016), 17-31.

[12] Gneezy, U. Deception: The role of consequences. American Econ. Review 95, 1 (2005), 384-394.

[13] HAYEL, Y., AND ZHU, Q. Attack-aware cyber insurance for risk sharing in computer networks. In Decision and Game Theory for Security. Springer, 2015, pp. 22-34.

[14] HaYel, Y., AND ZHU, Q. Epidemic protection over heterogeneous networks using evolutionary poisson games. IEEE Transactions on Information Forensics and Security 12, 8 (2017), 1786-1800.

[15] НoRÁк, K., ZHU, Q., AND BošANskỲ, B. Manipulating adversary?s belief: A dynamic game approach to deception by design for proactive network security. In International Conference on Decision and Game Theory for Security (2017), Springer, pp. 273-294.

[16] Huang, L., Chen, J., And Zhu, Q. A large-scale markov game approach to dynamic protection of interdependent infrastructure networks. In International Conference on Decision and Game Theory for Security (2017), Springer, pp. 357-376.

[17] Huang, L., AND Zhu, Q. Adaptive strategic cyber defense for advanced persistent threats in critical infrastructure networks. In ACM SIGMETRICS Performance Evaluation Review (2018).

[18] HuAng, L., AND ZHU, Q. Analysis and computation of adaptive defense strategies against advanced persistent threats for cyber-physical systems. In International Conference on Decision and Game Theory for Security (2018).

[19] HuANG, L., AND ZHu, Q. Adaptive strategic cyber defense for advanced persistent threats in critical infrastructure networks. ACM SIGMETRICS Performance Evaluation Review 46, 2 (2019), 52-56.

[20] HuAng, L., AND ZHu, Q. Dynamic bayesian games for adversarial and defensive cyber deception. In Autonomous Cyber Deception: Reasoning, Adaptive Planning, and Evaluation of HoneyThings, E. Al-Shaer, J. Wei, K. W. Hamlen, and C. Wang, Eds. Springer International Publishing, Cham, 2019, pp. 75-97.

[21] Jajodia, S., Ghosh, A. K., Swarup, V., Wang, C., AND Wang, X. S. Moving target defense: creating asymmetric uncertainty for cyber threats, vol. 54 . Springer Science \& Business Media, 2011.

[22] Manshaei, M. H., Zhu, Q., Alpcan, T., Bacşar, T., and Hubaux, J.-P. Game theory meets network security and privacy. ACM Computing Surveys (CSUR) 45, 3 (2013), 25.

[23] Miao, F., Zhu, Q., Pajic, M., and Pappas, G. J. A hybrid stochastic game for secure control of cyber-physical systems. Automatica 93 (2018), 55-63.

[24] Moghaddam, M. M., Manshaei, M. H., and Zhu, Q. To trust or not: A security signaling game between service provider and client. In Decision and Game Theory for Security. Springer, 2015, pp. 322-333.

[25] Pawlick, J. A Systems Science Perspective on Deception for Cybersecurity in the Internet of Things. $\mathrm{PhD}$ thesis, 2018. Copyright - Database copyright ProQuest LLC; ProQuest does not claim copyright in the individual underlying works; Last updated - 2018-08-09.

[26] Pawlick, J., Chen, J., ANd Zhu, Q. istrict: An interdependent strategic trust mechanism for the cloud-enabled internet of controlled things. IEEE Transactions on Information Forensics and Security (2018).

[27] Pawlick, J., Colbert, E., ANd Zhu, Q. A game-theoretic taxonomy and survey of defensive deception for cybersecurity and privacy. arXiv preprint arXiv:1712.05441 (2017).

[28] Pawlick, J., Colbert, E., ANd Zhu, Q. Modeling and analysis of leaky deception using signaling games with evidence. IEEE Transactions on Information Forensics and Security (2018). 
[29] Pawlick, J., Farhang, S., And Zhu, Q. Flip the cloud: Cyber-physical signaling games in the presence of advanced persistent threats. In Decision and Game Theory for Security. Springer, 2015, pp. 289-308.

[30] PAwlick, J., AND Zhu, Q. Deception by design: evidence-based signaling games for network defense. arXiv preprint arXiv:1503.05458 (2015).

[31] PAwLick, J., AND ZHU, Q. A Stackelberg game perspective on the conflict between machine learning and data obfuscation. IEEE Intl. Workshop on Inform. Forensics and Security (2016)

[32] PAwlick, J., AND ZHU, Q. A Stackelberg game perspective on the conflict between machine learning and data obfuscation. In Information Forensics and Security (WIFS), 2016 IEEE International Workshop on (2016), IEEE, pp. 1-6.

[33] PAwlick, J., AND ZHU, Q. Strategic trust in cloud-enabled cyber-physical systems with an application to glucose control. IEEE Transactions on Information Forensics and Security 12, 12 (2017), 2906-2919.

[34] Rass, S., Alshawish, A., Abid, M. A., Schauer, S., Zhu, Q., And de Meer, H. Physical Intrusion Games - Optimizing Surveillance by Simulation and Game Theory. IEEE Access (2017), 1.

[35] RAss, S., AND ZHU, Q. GADAPT: A Sequential Game-Theoretic Framework for Designing Defense-in-Depth Strategies Against Advanced Persistent Threats. In Decision and Game Theory for Security, Q. Zhu, T. Alpcan, E. Panaousis, M. Tambe, and W. Casey, Eds., vol. 9996 of Lecture Notes in Computer Science. Springer International Publishing, Cham, 2016, pp. 314-326.

[36] Vrij, A., Mann, S. A., Fisher, R. P., Leal, S., Milne, R., And Bull, R. Increasing cognitive load to facilitate lie detection: The benefit of recalling an event in reverse order. Law and Human Behavior 32, 3 (2008), 253-265.

[37] XU, Z., AND ZHU, Q. A cyber-physical game framework for secure and resilient multi-agent autonomous systems. In Decision and Control (CDC), 2015 IEEE 54th Annual Conference on (2015), IEEE, pp. 5156-5161.

[38] Xu, Z., AND ZHu, Q. Cross-layer secure cyber-physical control system design for networked 3d printers. In American Control Conference (ACC), 2016 (2016), IEEE, pp. 1191-1196.

[39] Xu, Z., And Zhu, Q. A Game-Theoretic Approach to Secure Control of Communication-Based Train Control Systems Under Jamming Attacks. In Proceedings of the 1st International Workshop on Safe Control of Connected and Autonomous Vehicles (2017), ACM, pp. 27-34.

[40] Xu, Z., AND ZHu, Q. Secure and practical output feedback control for cloudenabled cyber-physical systems. In Communications and Network Security (CNS), 2017 IEEE Conference on (2017), IEEE, pp. 416-420.

[41] YuAN, Y., ZHu, Q., Sun, F., WANG, Q., AND BASAR, T. Resilient control of cyberphysical systems against denial-of-service attacks. In Resilient Control Systems (ISRCS), 2013 6th International Symposium on (2013), IEEE, pp. 54-59.

[42] Zhang, N., Yu, W., Fu, X., AND DAs, S. K. gPath: A game-theoretic path selection algorithm to protect tor's anonymity. In Decision and Game Theory for Security (2010), Springer, pp. 58-71.

[43] Zhang, R., ANd Zhu, Q. Attack-Aware Cyber Insurance of Interdependent Computer Networks.

[44] Zhang, R., Zhu, Q., AND Hayel, Y. A Bi-Level Game Approach to Attack-Aware Cyber Insurance of Computer Networks. IEEE fournal on Selected Areas in Communications 35, 3 (2017), 779-794.

[45] Zhang, T., Huang, L., PAwlick, J., AND Zhu, Q. Game-theoretic analysis of cyber deception: Evidence-based strategies and dynamic risk mitigation. arXiv preprint arXiv:1902.03925 (2019)

[46] Zhang, T., AND ZHu, Q. Dynamic differential privacy for ADMM-based distributed classification learning. IEEE Transactions on Information Forensics and
Security 12, 1 (2017), 172-187.

[47] ZhANG, T., AND ZHu, Q. Strategic defense against deceptive civilian gps spoofing of unmanned aerial vehicles. In International Conference on Decision and Game Theory for Security (2017), Springer, pp. 213-233.

[48] Zhang, T., AND ZHu, Q. Distributed privacy-preserving collaborative intrusion detection systems for vanets. IEEE Transactions on Signal and Information Processing over Networks 4, 1 (2018), 148-161.

[49] Zhu, Q., AND BAŞAR, T. Dynamic policy-based ids configuration. In Decision and Control, 2009 held jointly with the 2009 28th Chinese Control Conference. CDC/CCC 2009. Proceedings of the 48th IEEE Conference on (2009), IEEE, pp. 8600-8605.

[50] ZHU, Q., AND BASAR, T. Game-theoretic approach to feedback-driven multi-stage moving target defense. In International Conference on Decision and Game Theory for Security (2013), Springer, pp. 246-263.

[51] Zhu, Q., Bushnell, L., AND BASAR, T. Game-theoretic analysis of node capture and cloning attack with multiple attackers in wireless sensor networks. In Decision and Control (CDC), 2012 IEEE 51st Annual Conference on (2012), IEEE, pp. 3404-3411.

[52] Zhu, Q., Clark, A., Poovendran, R., and Basar, T. Deceptive routing games. In Decision and Control (CDC), 2012 IEEE 51st Annual Conference on (2012), IEEE, pp. 2704-2711.

[53] Zhu, Q., Clark, A., Poovendran, R., And Basar, T. Deployment and exploitation of deceptive honeybots in social networks. In Decision and Control (CDC), 2013 IEEE 52nd Annual Conference on (2013), IEEE, pp. 212-219.

[54] Zhu, Q., Fung, C., Boutaba, R., ANd BAŞAR, T. A game-theoretical approach to incentive design in collaborative intrusion detection networks. In Game Theory for Networks, 2009. GameNets' 09. International Conference on (2009), IEEE, pp. 384-392.

[55] Zhu, Q., Fung, C., Boutaba, R., and Başar, T. Guidex: A game-theoretic incentive-based mechanism for intrusion detection networks. Selected Areas in Communications, IEEE fournal on 30, 11 (2012), 2220-2230.

[56] Zhu, Q., Gunter, C. A., AND BAsAR, T. Tragedy of anticommons in digital right management of medical records. In HealthSec (2012).

[57] Zhu, Q., Li, H., HAN, Z., AND BASAR, T. A stochastic game model for jamming in multi-channel cognitive radio systems. In ICC (2010), pp. 1-6.

[58] Zhu, Q., AND Rass, S. Game theory meets network security: A tutorial. In Proceedings of the 2018 ACM SIGSAC Conference on Computer and Communications Security (2018), ACM, pp. 2163-2165.

[59] Zhu, Q., Tembine, H., AND BAŞAR, T. Heterogeneous learning in zero-sum stochastic games with incomplete information. In Decision and Control $(C D C)$, 2010 49th IEEE Conference on (2010), IEEE, pp. 219-224.

[60] Zhu, Q., Tembine, H., ANd BAsAR, T. Network security configurations: A nonzerosum stochastic game approach. In American Control Conference (ACC), 2010 (2010), IEEE, pp. 1059-1064.

[61] Zhu, Q., Tembine, H., AND Basar, T. Hybrid learning in stochastic games and its applications in network security. Reinforcement Learning and Approximate Dynamic Programming for Feedback Control (2013), 305-329.

[62] Zhu, Q., Yuan, Z., Song, J. B., Han, Z., AND BASAR, T. Dynamic interference minimization routing game for on-demand cognitive pilot channel. In Global Telecommunications Conference (GLOBECOM 2010), 2010 IEEE (2010), IEEE, pp. 16

[63] ZhU, Q., YuAN, Z., Song, J. B., HAN, Z., AND BAşAR, T. Interference aware routing game for cognitive radio multi-hop networks. Selected Areas in Communications, IEEE Journal on 30, 10 (2012), 2006-2015. 